\title{
Short Term Outcomes in Patients Undergoing Aseptic Total Knee Arthroplasty Revision
}

\author{
ErhanSukur, MD ${ }^{1 *}$, Yusuf Ozturkmen, M.D² \\ ${ }^{1}$ Department of Orthopaedics and Traumatology, Sakarya Training and Research Hospital, Sakarya, Turkey \\ ${ }^{2}$ Department of Orthopaedics and Traumatology, Istanbul Training and Research Hospital, Istanbul, Turkey \\ *Corresponding Author:Dr Erhan Sukur,Department of Orthopaedics and Traumatology, Sakarya \\ Training and Research Hospital, Sakarya, Turkey, E-mail: erhan_sukur@hotmail.com
}

\begin{abstract}
Background: A significant number of patients eventually face the prospect of revision knee arthroplasty. Hence, it is of high importance to assess postoperative outcomes of revision total knee arthroplasty.

Objective: The purpose of the present study was to evaluate the indications and outcomes of patients who underwent revision total knee arthroplasty for aseptic reasons.

Method: Thirty-five knees of 34 patients that underwent aseptic revision total knee arthroplasty between April 2003 and March 2008 were retrospectively reviewed. In order to exclude infection, physical examination, laboratory tests and nuclear medicine examination (Tc 99m) were performed. The patients were evaluated with respect to age, gender, cause of primary and revision TKA and prosthesis types they were currently using. Clinical and functional assessment were performed using the American Knee Society questionnaire. Radiographic examination included weight-bearing anteroposterior and lateral knee views and tangential radiographs of the patellofemoral joint carried out by assessing loosening, migration and any radiolucency.
\end{abstract}

Results: Revision knee arthroplasty surgery was performed in 21 knees (60\%) for aseptic loosening, in 11 knees (31.4\%) for extensor mechanism problems and in 3 knees (8.5\%) for the diagnosis of periprosthetic fracture. The preoperative and postoperative mean clinical knee scores were 48.8 (27-78) and 80.4 (43-92), respectively $(p<0.001)$. The preoperative and postoperative mean functional knee scores were 38.3 (15-75) and $73.4(45-100)$, respectively $(p<0.001)$.

Conclusion: Aseptic loosening is the most common reason for aseptic revision TKA. Satisfactory clinical outcomes may be obtained when stable long-term fixation and anatomic relationships of the knee are restored.

\section{INTRODUCTION}

Total knee arthroplasty (TKA) has been known to be effective for patients with knee osteoarthritis, a condition that impairs the quality of life of patients by causing pain, limits in the range of motion, and deformity in the knee (1). According to the latest worldwide joint registries, revision for total knee arthroplasty is on average $12 \%$ over a period of 10 years and dropped due to the use of advanced implant technology, surgical techniques, and measures to prevent infection $(2,3)$. However, the absolute number of TKA revisions have shown a steady increase due to the greater need for primary TKA (4). To perform a successful revision total knee arthroplasty, certain steps must be followed. Each step should be reviewed in detail and along with a pre-operative planning, techniques for the surgical approach and component removal, and the selection of appropriate prosthetic components should be discussed. Possible technical difficulties such as bone loss, ligamentous instability, and management of the extensor mechanism are also important points to be achieved for a successful revision total knee arthroplasty (5).

A significant number of patients eventually face the prospect of revision knee arthroplasty due to infection or aseptic reasons, such as mechanical wear, aseptic loosening, instability, malalignment, and periprosthetic fractures (6, 7). Unfortunately, the overall outcome is not as desirable as those of primary arthroplasty $(2,7)$. There are few studies that have evaluated the 
postoperative complications of revision TKA and limited data are available on revision TKA. Hence, it is of high importance to assess postoperative outcomes of revision TKA (8). Revisions after knee arthroplasty are expected to increase, and the epidemiology of failure mechanisms is changing as new implants, technology, and surgical techniques evolve. Knowledge of the etiology of failure mechanisms is of paramount importance for delivery of appropriate care. To improve the economic burden of aseptic complications of TKA in our institution, the best way was to understand the etiology of TKAs that needed revisions and to evaluate the methods used for revision. The purpose of the present study was to provide a detailed analysis of the indications and outcomes of patients at a single institution for whom revision TKA was performed for aseptic reasons and to compare the results with those reported by similar investigations in the light of the literature.

\section{MATERIALS AND METHODS}

This study was approved by a clinical research ethics board and executed in accordance with the Helsinki Declaration. 50 patients (55 knees) that underwent revision TKA at our institution between April 2003 and March 2008 were retrospectively reviewed. Thirty-five knees of 34 patients ( 29 females- 30 knees and 5 males) in which aseptic revision TKA was performed were included in the study. In order to exclude infection, physical examination, laboratory tests (C-reactive protein, erythrocyte sedimentation rate, WBC count) and nuclear medicine examination (Tc 99m) were performed. In suspected cases, joint aspiration was performed under sterile conditions and gram staining and bacteriological examination of the fluid were Table1: Demographic Data of the Patients performed. The patients were evaluated with respect to age, gender, cause of primary and revision TKA and prosthesis types they were currently using. Old incision scars, joint range of motion, presence or absence of ligament failures and presence or absence of bone defects on the obtained radiographs and the complications were also noted. Clinical and functional assessment were performed for all patients using the American Knee Society (AKS) questionnaire preoperatively and at last follow-up examinations. Radiographic examination included weight-bearing anteroposterior and lateral knee views and tangential radiographs of the patellofemoral joint carried out by assessing loosening, migration and any radiolucency around the femur, tibia and patella.

Statistical evaluation in the proposed study was performed using the SPSS 15.0 application for Windows (SPSS Inc., Chicago, IL, USA). Descriptive statistics were provided to represent the mean and standard deviation for numeric variables. Statistical evaluation of scores was calculated using 95\% confidence intervals and parametric paired t-tests. The significance level for each of the above tests was set at 0.001 .

\section{ReSults}

Demographic data of revision TKA Patients are reflected in Table 1. The AKS clinical and functional scores were both significantly improved postoperatively $(\mathrm{p}<0.001)$. The mean knee flexion angle and flexion contracture measurements demonstrated significant improvement as well $(\mathrm{p}<0.001)$ and presented in Table 2.

\begin{tabular}{|c|c|}
\hline \multicolumn{2}{|c|}{ Gariable } \\
\hline \multicolumn{1}{|c|}{ Gender (n), (\%) } \\
\hline Female & $29(85.2)$ \\
\hline Male & $5(14.79)$ \\
\hline Left & $15(42.8)$ \\
\hline Right & $18(51.4)$ \\
\hline Bilateral & $1(2.8)$ \\
\hline Age at primary TKA, Mean \pm SD (min-max) $(\%)$ & $58.5 \pm 7.6(49-73)$ \\
\hline Age at revision TKA, Mean \pm SD (min-max) & $66.8 \pm 8.4(52-88)$ \\
\hline Time between primary and revision TKA (months) & $72.4 \pm 2.6(60.5-92.4)$ \\
\hline Duration of follow-up (months) & $22.3 \pm 1.2(12-72)$ \\
\hline Cause of primary TKA (n*), (\%) & $29(82.8)$ \\
\hline Primary osteoarthritis & $3(8.5)$ \\
\hline Rheumatoid arthritis & $3(8.5)$ \\
\hline Post-traumatic arthrosis &
\end{tabular}




\begin{tabular}{|c|c|}
\hline \multicolumn{2}{|c|}{ Cause of revision TKA $\left(\mathrm{n}^{*}\right),(\%)$} \\
\hline Aseptic loosening & $21(60)$ \\
\hline Extensor mechanism problems & $3(31.4)$ \\
\hline Peri-prosthetic fractures & \\
\hline $\boldsymbol{n}:$ Number of patients \\
\hline $\boldsymbol{n}^{*}:$ number of cases
\end{tabular}

Table2: The Clinical and Functional Outcomes

\begin{tabular}{|c|c|c|}
\hline & Preoperatively & Postoperatively \\
\hline *AKS Clinical score, Mean \pm SD & $48.8 \pm 10$ & $80.4 \pm 8.9$ \\
\hline *AKS Functional score, Mean \pm SD & $38.3 \pm 13.8$ & $73.4 \pm 12.2$ \\
\hline \multicolumn{2}{|c|}{ Measurements of the knee joint (degree) } \\
\hline Knee flexion, Mean \pm SD & $75.1 \pm 15.7$ & $97 \pm 12.6$ \\
\hline \multicolumn{2}{|c|}{ Knee flexion contracture, Mean \pm SD } & $6.8 \pm 12.4$ \\
\hline \multicolumn{2}{|c|}{ *American Knee Society } \\
\hline
\end{tabular}

Loosening of tibial and femoral components were observed and replaced in all patients with the diagnosis of aseptic loosening. Long-stem components and cemented fixation were preferred in all cases (Figure 1).In 15 of these 21 patients, patellar component had also been implanted during the first surgery. No loosening was observed and patellar components were preserved. six patients who had not received patellar component in the first operation were implanted with a patellar component during the revision surgery. A thicker insert compared to the existing one was had to be used in 18 of these 21 patients. The previous anterior longitudinal incision was used in all cases. The joint was reached using medial parapatellar approach except in two patients. These two patients had lateral subluxation of the patella during the preoperative period. In these two patients, lateral parapatellar approach was preferred and medial plication was performed at the end of the operation. In 11 of the 32 cases with both aseptic loosening and extensor mechanism problems, tibial tubercle osteotomy was performed in addition to the standard surgical approaches in order to expose and remove the components. In all patients in whom tibial tubercle osteotomy was performed, the preoperative total range of motion in the knee joint was less than 75 degrees and the patella could not be turned down laterally by standard surgical approaches. Combinations of lag screws and cerclage wires were used for the fixation of the tibial tubercle at the end of the operation (Figure 2).

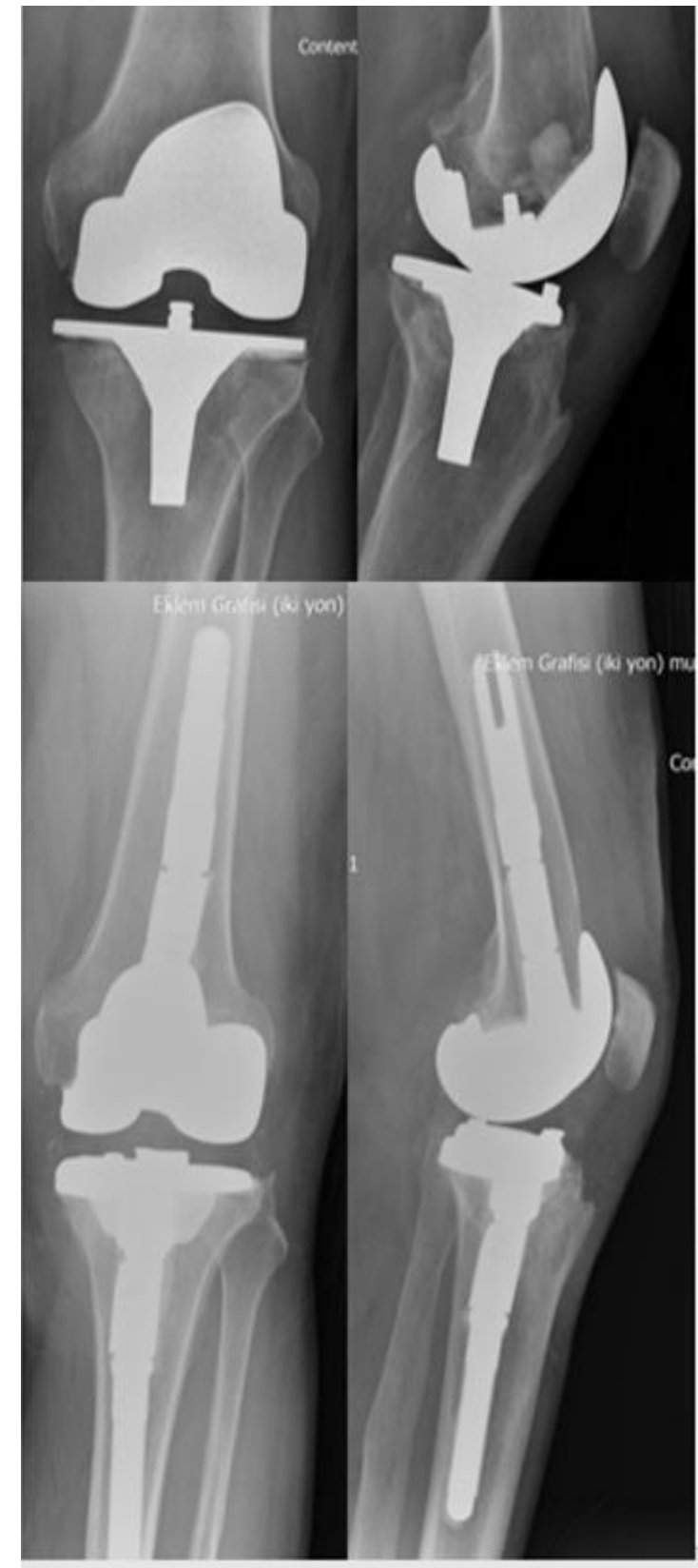

Figure1: Aseptic Loosening of the Tibial and Femoral Component Revised with Long Cementless Tibial and Femoral Stems 


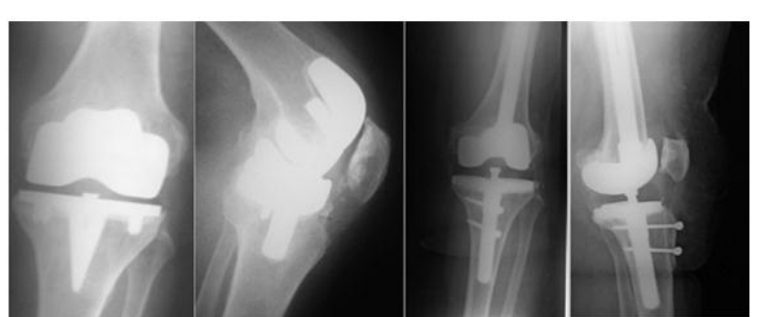

Figure2: Tibial Tubercule Osteotomy was Performed and Fixated with Two Lag Screws Fort he Adequate Expojure

In five patients with a preoperative total range of motion less than 60 degrees, fixation was achieved by shifting the tibial tubercle superiorly. Iatrogenic patellar tendon rupture occurred in one of the patients during the removal of the components. After the primary repair of the tendon, the repair was reinforced with a cerclage wire passed through the lower pole of the patella and the tibial tubercle. The problem was patellofemoral instability in eight of 11 patients undergoing revision for extensor mechanism problems. Out of the remaining three patients, the reason for revision was patellar tendon rupture in two patients and patellar fracture in one patient. Since primary repair was not possible in the patients with patellar tendon rupture, hamstring autografts were used instead of patellar tendon and reinforced the repair with cerclage wire. The patient with patellar fracture had a history of high-energy trauma. Fracture fixation was achieved by using zugurtung technique in this patient who did not have patellar component. Out of the three patients who underwent revision for periprosthetic fracture, the fracture was in the femoral supracondylar region in one patient, in the medial femoral condyle in one patient and in the patella in the remaining patient. The periprosthetic fractures at the femoral site were classified according to the Lewis and Rorabeck Classification system as type 2 and type 3 respectively (9). Component stability was not impaired in the patient who had supracondylar femur fracture. Fixation was performed using retrograde femoral nail (Figure $3)$.

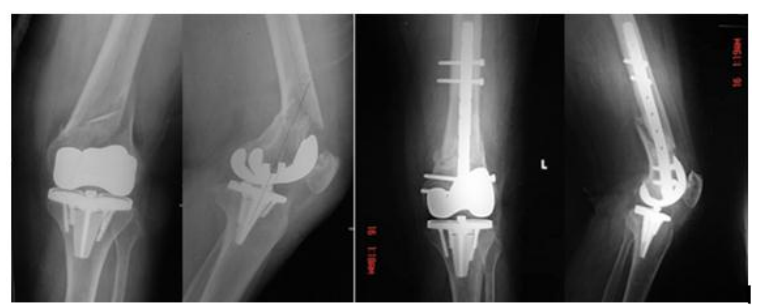

Figure3:Suprcondyler Periprosthetic Femur Fracture Treated with Retromedullar Nailing
In the patient with medial femoral condyle fracture, the femoral component was replaced with the long-stem component and the fractured condyle was fixed to the metaphysis using lag screws. In the patient with periprosthetic patellar fracture, it was found out during the operation that patellar component stability was not lost and fracture fixation was performed using Magnusson technique. Out of 16 knees with bone defects that would cause instability and/or change the joint level before the operation or after the components were removed during the operation, metal wedge and blocks were used to fill the bone defects in the posterior condyles of the femur and tibial plateau in 12 knees and in only the tibial plateau in four knees. Active extension loss was measured in 12 knees preoperatively, while it was measured in four knees postoperatively as 10 degrees on average. These four knees belonged to the patients undergoing revision for patellar tendon rupture and patellar fracture. Three patients developed superficial infections after revision surgery. All of these three patients could be treated with antibiotherapy and no debridement was required. In two patients, a re-revision was had to be performed due to deep infection. Twostage reimplantation was preferred. Reimplantation was performed after a mean period of 126 days following the removal of the implants and spacer placement.

\section{DISCUSSION}

As in all other specialties, with the advancing technology, the complications associated with knee arthroplasty started to be understood and treated better $(2,8)$. Existing bone defects, ligament imbalances, fixation problems, the difficulty of surgical exposure and associated complication risks and high infection rates are enough to make surgeons very cautious and worried about revision TKA (10). There are few studies in the literature that report the distribution of aseptic revisions according their etiologies. Among these, Friedman et al. reported in their series that the reason for aseptic revision was aseptic loosening in $73 \%$, extensor mechanism problems in $13 \%$, instability in $10 \%$ and other reasons in $4 \%$ of the cases (11).In our series, the rates are $57 \%$ for aseptic loosening, $34 \%$ for extensor mechanism dysfunction and $8.5 \%$ for prosthetic fracture and these rates are similar to the literature

The complication rate of revision TKA is quite higher when compared to that of primary TKA. In the literature, the greatest emphasis has been 
placed on infections among the complications of revision TKA. Naturally, the infection rates after aseptic revisions are significantly lower than the infection rates seen in patients undergoing revision for septic reasons (12). Infection rates after aseptic revisions reported by Goldberg, the HSS team and Insall were $4.5 \%, 5 \%$ and $9 \%$, respectively $(12,13)$. In our series, the rate of infection following aseptic revisions was $6.06 \%$ and consistent with the literature. Other complications may include healing problems, aseptic loosening, limitation of motion and instability. The rates of these types of complications after secondary revision reported by Goldberg, Bertin and Jacobs were $15.3 \%, 22 \%$ and $10 \%$, respectively $(13,14,15)$. It is $20 \%$ in our series. The patients live in different regions of our country and this makes their routine controls harder. Therefore, small problems which can be easily revised in shortterm, are encountered as complicated problems in the long-term. The rate of factors that complicate surgery, such as bone defects and ligament imbalances were high in many of the patients undergoing revision surgery and we believe that these factors contributed to the secondary revision rate which was at the higher limit of the range reported in the literature $(11,14)$. The objective outcomes and patient satisfaction for revision TKA are lower when compared to primary TKA (16).Ching-Jen Wang et al. conducted a study comparing clinical and functional outcomes and patient satisfaction between patients who underwent revision for aseptic or septic reasons (17).In this study, they reported that patient satisfaction and joint range of motion were higher after aseptic revisions, while there was no significant difference in terms of pain and functional scores. In the present study, the mean clinical knee score was 86.5 and the mean functional knee score was 68.6 in patients who underwent aseptic revision. It was also observed that, the mean knee score assessed using the AKS scoring system increased from the preoperative 48.89 points to 80.4 points postoperatively. Similarly, the mean functional score was 38.3 preoperatively, while it was found to be 73.4 postoperatively. The increases in clinical and functional scores are statistically significant $(\mathrm{p}<0.001)$.

In studies on all revisions, including those that were performed for both aseptic and septic reasons, total knee range of motion was reported to be 84 degrees by Goldberg and 100 degrees by Bertin (13,15).In their study evaluating only aseptic revisions Ching-Jen Wang et al. reported that it was 90.3 degrees on average (16).In this study, the mean angle of knee flexion was 75.1 degrees preoperatively, which increased to 97 degrees postoperatively $(\mathrm{p}<0.001)$.The mean flexion contracture angle was 6.8 degrees preoperatively and decreased to the postoperative 1.5 degrees $(\mathrm{p}<0.002)$.It was observed that these postoperative joint range of motion values were superior when compared to the values reported in many studies in the literature. Due to the life style of our population, more knee range of motion is required (sitting cross-legged, performing namaz, allaturca toilet, eating on the floor etc.) We attribute the lower patient satisfaction level compared to the series in the literature with similar range of motion to these factors.

The designs and types of prostheses used in revision surgery vary widely depending on the individual difficulties in each patient (17). Prosthesis selection varies depending on the patient, the technique preferred by the surgeon and available prosthesis options. However, the general approach includes using long-stem prostheses in the knees with bone defects and constrained prostheses are used for the knees with ligament imbalance (18).Constrained prostheses were used in three of the patients. One of these patients was the patient who developed an infection after revision and had associated serious bone defect and the other two patients were the ones in whom multiple revisions were had to be performed for the diagnosis of persistent instability.

The most common reason for aseptic revisions is aseptic loosening. The most important cause of aseptic loosening is surgical technical mistakes resulting in malalignment and malposition (19).Aseptic loosening is most commonly seen in the tibial component. It is reported that the most ideal placement of the components is at 5-15 degrees of valgus $(19,20)$.In these cases, lower extremity orthographies were taken and evaluated according to the AKS radiological assessment form. Six of the patients had postoperative lower extremity alignment values outside the ideal range and two of these patients underwent secondary revision surgery for the diagnosis of instability. Some factors determining aseptic loosening include the quality of fixation, the activity level and weight of the patient and 
osteolysis (21).We do not have enough documentation to determine how effective these factors, which promote aseptic loosening, are on the need for revision. A particular limitation of this study is that computerized tomography was not used to assess rotational lower extremity problems.

In the literature, the most important factor determining long-stem use is the remaining amount of quality bone after the removal of the components both on tibial and femoral sides. Quality bone refers to the bone tissue that allows adequate fixation (22).Jazrawi et al., in their cadaveric study, investigated the effects of stem length and diameter on the stress transfer to the tibia and reported that the stress that develops in the tibia decreases in parallel with an increase in the stem diameter and length. They indicated that the long-term potential problems that may develop in the tibial component decrease accordingly (22).Longstem was also used on both tibial and femoral side in all of these cases in whom revision TKA was performed for aseptic loosening. The low rate of radiolucent lines in the follow-ups is attributed to the routine use long-stem. Based on experience, quality of fixation is one of the most important factors that decrease the risk of aseptic loosening particularly after revision operations. In all cases undergoing revision there is some degree of bone loss. We believe that even small amounts of bone loss may put fixation at risk.

Patellar instability represents the largest group among extensor mechanism problems following TKA (23). Among the patients in whom we performed revision for extensor mechanism problems, eighth $(72 \%)$ had patellar instability, one $(9 \%)$ had patellar fracture and two $(18 \%)$ had patellar tendon rupture as the primary diagnosis. Patellar tendon injuries occurring during exposure and when the patella is turned down laterally during revision surgery have been reported in the literature at variable rates (24).Halder performed tibial tubercle osteotomy in 67 RKAS patients and encountered pseudoarthrosis in only one case, consistent with performing the surgical technique correctly. Tibial tubercle osteotomy was defined as a safe and effective technique in patients with contracture, in whom the knee cannot be opened with the standard medial and lateral parapatellar approach $(25,26)$. Tibial tubercle osteotomy technique was also used and the formed bone block was turned over laterally to avoid hard-totreat complications in the patients. Lateral soft tissue support was preserved during osteotomy. We used combinations of cerclage wires and screw fixation for the fixation of the osteotomized bone block. We did not deem it necessary to apply casts or braces during the postoperative rehabilitation. Flexion and total joint ranges of motion were consistent with the literature.

\section{CONCLUSiON}

Revision TKA is a complex surgery involving high risks. It is less successful and satisfactory compared to primary surgery. One of the important factors affecting the outcome of revision surgery is the time between the onset of complaints of the patient and the surgery. In patients who present late, development of and increase in bone loss, addition of instability and osteopenia, and muscle atrophies associated with disuse complicate the planned surgical technique and put the quality of fixation at risk. In such cases long-stem components should be preferred and implantation should be performed with press-fit technique. Various types of prostheses should be made available in the operating room. Strict adherence to the principles is essential for minimizing complications.

ACKNOWLEDGEMENTS: The authors thank Mustafa Caniklioglu, MD, for his contribution to this study.

\section{REFERENCES}

[1] Harmelink KEM, Zeegers AVCM, Hullegie W, Hoogeboom TJ, Nijhuis-van der Sanden MWG, Staal JB. Are There Prognostic Factors for One-Year Outcome After Total Knee Arthroplasty? A Systematic Review.J Arthroplasty. 2017 Dec;32(12):3840-3853.e1. doi: 10.1016/j.arth.2017.07.011. Epub 2017 Aug 1. PMID: 28927646

[2] Labek G, Thaler M, Janda W, Agreiter M, Stöckl B. Revision rates after total joint replacement: cumulative results from worldwide joint register datasets. J Bone Joint Surg Br 2011;93:293-7.

[3] Sibanda N, Copley LP, Lewsey JD, Borroff M, Gregg P, MacGregor AJ, Pickford M, Porter M, Tucker K, van der Meulen JH; Steering Committee of the National Joint Registry (NJR) for England and Wales. Revision rates after primary hip and knee replacement in England between 2003 and 2006. PLoS Med. 2008;5:e179.

[4] Kamath AF, Lee GC, Sheth NP, Nelson CL, Garino JP, Israelite CL. Prospective results of uncemented tantalum monoblock tibia in total 
knee arthroplasty: minimum 5-year follow-up in patients younger than 55 years. $\mathrm{J}$ Arthroplasty 2011; 26:1390-5.

[5] Vasarhelyi EM, MacDonald SJ, Della Valle CJ, Vail TP. Let's do a revision total knee arthroplasty. Instr Course Lect. 2014;63:23951.

[6] Saleh KJ, Rand JA, McQueen DA. Current status of revision total knee arthroplasty: how do we assess results? J Bone Joint Surg Am 2003; 85-A Suppl 1:S18-S20.

[7] Tekin B, Unver B, Karatosun V. Expectations in patients with total knee arthroplasty. Acta Orthop Traumatol Turc 2012; 46:174-80

[8] Lovelock TM, Broughton NS. Follow-up after arthroplasty of the hip and knee: are we overservicing or under-caring? Bone Joint J. 2018 Jan;100-B(1):6-10. doi: 10.1302/0301620X.100B1.BJJ-2017-0779.R1. Review.

[9] Rorabeck CH, Angliss RD, Lewis PL. Fractures of the femur, tibia, and patella after total knee arthroplasty: decision making and principles of management. Instr Course Lect. 1998;47:44958.

[10] Sharkey PF, Lichstein PM, Shen C, Tokarski AT, Parvizi J. Why are total knee arthroplasties failing today--has anything changed after 10 years? J Arthroplasty. 2014 Sep;29(9):1774-8. doi: 10.1016/j.arth.2013.07.024. Epub 2014 Jul 5.

[11] Friedman RJ, Hirst P, Poss R, Kelley K, Sledge $\mathrm{CB}$. Results of revision total knee arthroplasty performed for aseptic loosening. Clin Orthop Relat Res 1990; 255:235-41.

[12] Insall JN. Revision of aseptic failed total knee arthroplasty. Insall JN (ed). Surgery of the knee. 2nd edition. New York, Churchill Livingstone Inc. 935, 1993.

[13] Bertin KC. Evaluation of the failed total knee arthroplasty. Callaghan JJ. Hip and knee reconstruction. American academy of orthopaedic surgeons 317, 1995.

[14] Wood GC, Naudie DD, MacDonald SJ, McCalden RW, Bourne RB. Results of press-fit stems in revision knee arthroplasties. Clin Orthop Relat Res 2009; 467:810-7.

[15] Goldberg VM, Figgie MP, Figgie HE 3rd, Sobel M. The results of revision total knee arthroplasty. Clin Orthop Relat Res 1988; 226:86-92.

[16] Wang CJ, Hsieh MC, Huang TW, Wang JW, Chen HS, Liu CY. Clinical outcome and patient satisfaction in aseptic and septic revision total knee arthroplasty. Knee 2004; 11:45-9.

[17] Hwang SC, Kong JY, Nam DC, Kim DH, Park $\mathrm{HB}$, Jeong ST, Cho SH. Revision total knee arthroplasty with a cemented posterior stabilized, condylar constrained or fully constrained prosthesis: a minimum 2-year follow-up analysis. Clin Orthop Surg. 2010 Jun;2(2):112-20.

doi: 10.4055/cios.2010.2.2.112. Epub 2010 May 4.

[18] Conlisk N, Howie CR, Pankaj P. Computational modelling of motion at the bone-implant interface after total knee arthroplasty: The role of implant design and surgical fit. Knee. 2017 Oct;24(5):994-1005. doi: 10.1016/j.knee.2017.07.003. Epub 2017 Aug 1.

[19] Nedopil AJ, Howell SM, Hull ML. What mechanisms are associated with tibial component failure after kinematically-aligned total knee arthroplasty? Int Orthop. 2017 Aug;41(8):1561-1569. doi: 10.1007/s00264017-3490-6. Epub 2017 May 11.

[20] Jasper LL, Jones CA, Mollins J, Pohar SL, Beaupre LA. Risk factors for revision of total knee arthroplasty: a scoping review. BMC Musculoskelet Disord. 2016 Apr 26;17:182. doi: 10.1186/s12891-016-1025-8. Review.

[21] Rozkydal Z, Janík P, Janícek P, Kunovský R. [Revision knee arthroplasty due to aseptic loosening]. [Article in Czech] Acta Chir Orthop Traumatol Cech 2007; 74:5-13.

[22] Jazrawi LM, Bai B, Kummer FJ, Hiebert R, Stuchin SA. The effect of stem modularity and mode of fixation on tibial component stability in revision total knee arthroplasty. J Arthroplasty 2001; 16:759-67.

[23] Zhamilov V, Karatosun V, Kalkan S, Unver B, Gunal I. Evaluation of Extensor Mechanism in Revision Knee Arthroplasty. J Arthroplasty. 2017 Aug;32(8):2484-2486. doi: 10.1016/j.arth.2017.03.028. Epub 2017 Mar 22.

[24] Lamberti A, Balato G, Summa PP, Rajgopal A, Vasdev A, Baldini A. Surgical options for chronic patellar tendon rupture in total knee arthroplasty. Knee Surg Sports Traumatol Arthrosc. 2016 Nov 5.

[25] Halder AM. Tibial tubercle osteotomy. Open Orthop Traumatol 2012; 24:85-94.

[26] Clarke HD. Tibial tubercle osteotomy. J Knee Surg. 2003 Jan;16(1):58-61.

Citation:Erhan Sukur, MD, Yusuf Ozturkmen, M.D, Short Term Outcomes in Patients Undergoing Aseptic Total Knee Arthroplasty Revision, ARC Journal of Orthopedics. 2018; 3(1):11-17. doi:dx.doi.org/ 10.20431 /2456-0588.0301003.

Copyright: (C) 2018 Authors. This is an open-access article distributed under the terms of the CreativeCommonsAttributionLicense, which permits unrestricted use, distribution, and reproduction in any medium, provided the original author and source are credited. 